\title{
Inter-facility Transfer of Surgical Emergencies in a Developing Country: Effects on Management and Surgical Outcomes
}

\author{
Salma Khan · Hasnain Zafar • Syed Nabeel Zafar • \\ Naveed Haroon
}

Published online: 1 November 2013

(C) Société Internationale de Chirurgie 2013

\begin{abstract}
Objectives Outcomes of surgical emergencies are associated with promptness of the appropriate surgical intervention. However, delayed presentation of surgical patients is common in most developing countries. Delays commonly occur due to transfer of patients between facilities. The aim of the present study was to assess the effect of delays in treatment caused by inter-facility transfers of patients presenting with surgical emergencies as measured by objective and subjective parameters.

Methods We prospectively collected data on all patients presenting with an acute surgical emergency at Aga Khan University Hospital (AKUH). Information regarding demographics, social class, reason and number of transfers, and distance traveled were collected. Patients were categorized into two groups, those transferred to AKUH from another facility (transferred) and direct arrivals (nontransfers). Differences between presenting physiological parameters, vital statistics, and management were tested between the two groups by the chi square and $t$ tests.

Results Ninety-nine patients were included, 49 (49.5\%) patients having been transferred from another facility. The most common reason for transfer was "lack of satisfactory
\end{abstract}

\section{S. Khan}

Department of Surgery, Memon Medical Institute,

Karachi, Pakistan

S. Khan $(\bowtie)$

Flat \#47, Society Apartments, Block A, PECHS,

Karachi, Pakistan

e-mail: dr_salmakhan@yahoo.com

H. Zafar · S. N. Zafar · N. Haroon

Department of Surgery, Aga Khan University Hospital,

Karachi, Pakistan surgical care." There were significant differences in presenting pulse, oxygen saturation, respiratory rate, fluid for resuscitation, glasgow coma scale, and revised trauma score (all $p$ values $<0.001$ ) between transferred and nontransferred patients. In 56 patients there was a further delay in admission, and the most common reason was bed availability, followed by financial constraints. Three patients were shifted out of the hospital due to lack of ventilator, and 14 patients left against medical advice due to financial limitations. One patient died.

Conclusions Inter-facility transfer of patients with surgical emergencies is common. These patients arrive with deranged physiology which requires complex and prolonged hospital care. Patients who cannot afford treatment are most vulnerable to transfers and delays.

\section{Introduction}

According to World Health Organization estimates, approximately two billion people around the world have inadequate access to surgical care [1]. The disease control priorities project determined that surgical intervention may avert at least $11.2 \%$ of the global burden of disease by reducing disability and premature death [1-3]. The highest number of surgical disability-adjusted life years (DALYs) is recorded in South East Asia, and Africa has the highest ratio of surgical DALYs per 1,000 people [2].

Surgical care is considered to be an integral part of health systems worldwide [4]; still, delays due to multiple transfers of surgical patients are not uncommon, even in developed countries [5, 6]. Holena et al, [6] while assessing factors associated with outcomes in patients with necrotizing fasciitis, found inter-hospital transfers to be an independent risk factor for increased mortality. In a survey 
of surgical emergencies in Pakistan, Mushtaq et al [7] found that $28 \%$ of patients with acute abdomen presented initially to a center where surgical facilities were not available and therefore had to be transferred. Organized transportation is required for safe transfer of such patients to a surgical care facility. Unfortunately, the ambulance system is quite immature in Pakistan, despite the increasing demand for medical transport, chiefly because of the political situation [8,9]. Razzak et al [10] in a study on emergency medical services and mode of patient's transportation in Karachi, observed that the most common mode of transport to the emergency department (ED) was taxi $(53,58 \%)$, followed by private car $(21,23 \%)$.

Provision of essential surgical care remains inadequate in Pakistan, especially in rural and remote areas [11]. Patients are often transferred to far off urban tertiary care hospitals, resulting in delayed presentation [7, 11, 12]. These patients are at increased risk for metabolic, cardiovascular, renal, pulmonary, and septic complications before, during, and after the surgical intervention [13].

To the best of our knowledge, this region has never been subjected to any study by a local or international body relating to the fallout from delayed transfer of patients. Furthermore, there is a lack of data regarding the proportion or effect of inter-hospital transfer of patients vis-à-vis surgical emergencies. The aim of the present study was to assess the effect of delays in treatment on initial patient presentation, management, and subjective and objective outcomes on account of inter-facility transfers of patients with surgical emergencies.

Because the international literature is lacking in this respect, this study may prove to be pioneering work in objectively assessing outcomes of delayed transfer and its outcome. Pakistan, due to its geopolitical location and prevalent law-and-order situation needs special attention of international community.

\section{Methods}

We prospectively collected information from all consecutive patients aged 15 years or older presenting to the emergency department of the Aga Khan University Hospital (AKUH) for whom a surgical consult or a trauma call was generated from 1 to 15 May, 2012 [14].

Aga Khan University hospital (AKUH) is a 563-bed tertiary health institution situated in southeast Pakistan [15]. As a major referral center; the hospital receives patients with surgical problems from all over the country.

Data were collected by two trained surgical residents who also took part in the study design and development of the questionnaire. Informed consent was taken from the patient or an immediate family member able to make decisions for a patient in a critical condition. Confidentiality was maintained and no patient identifying information was recorded beyond patient discharge. No intervention was performed, and care was taken so that administration of the questionnaire would not hinder patient management in any way.

Data were collected from four distinct sources with a standardized questionnaire: (1) patients or patients' attendants (age, gender, social class variables, transfer status, number of transfers, reason for transfer, mode of transportation, distance traveled); (2) emergency department record (vitals at presentation, volume of fluid resuscitation); (3) surgical chief resident (change in American Society of Anesthesiologists [ASA] status, procedure); and (4) patient file at discharge (surgical procedure, need for invasive monitoring, length of hospital stay, intensive care unit [ICU] admission, delayed admission). "social class" was defined in accordance with the Ministry of Politics and Society, Islamabad 2008.We defined "delayed admission" if a patient remained in the Emergency Department (ED) longer than $4 \mathrm{~h}$ before being admitted to the hospital ward or intensive care unit (ICU) [16].

Patients were divided into two groups; those transferred to AKUH from another facility (transferred) and direct arrivals at AKUH (non-transferred). The effect of interfacility transfer was measured by objective and subjective parameters. Objective parameters included patient physiology at initial presentation to the ED (pulse, respiratory rate, oxygen saturation, resuscitation fluid requirements, Glasgow Coma Score [GCS], RTS) and hospital course (surgical procedure, need for invasive monitoring, length of hospital stay, ICU admission, and delayed admission). Subjective parameters included the surgical Chief Resident's assessment of change in ASA status, change in surgical procedure performed. Continuous variables such as age, total transfer time, and distance traveled were analyzed as means with standard deviations. Categorical variables such as gender, social class, ED presentation, number of transfers, etc. were described as proportion with percentages. Between-group comparisons were made with Student's $t$-test and the chi square test. Data were analyzed with SPSS version 17. A $p$ value less than 0.05 was considered significant.

\section{Results}

A total of 99 patients were included in the study. The demographic details are presented in Table 1. The mean age of transferred and non-transferred patients was comparable ( $43 \pm 13$ vs. $40 \pm 15$ years). Gender was evenly distributed, with $54.4 \%$ of the patients being male. The majority of patients belonged to middle to low social class $(88 \%)$. Acute intestinal obstruction $(12.1 \%)$, cholecystitis 
Table 1 Demographics and baseline information of patient's status on presentation

\begin{tabular}{|c|c|c|c|}
\hline Variable & $\begin{array}{l}\text { Transfer } \\
(n=49)\end{array}$ & $\begin{array}{l}\text { Non-transfer } \\
(n=50)\end{array}$ & $p$ value \\
\hline Age, years & $43 \pm 13$ & $40 \pm 15$ & 0.152 \\
\hline \multicolumn{4}{|l|}{ Gender } \\
\hline Male & $29(29.2 \%)$ & $25(25.2 \%)$ & \\
\hline Female & $20(20.2 \%)$ & $25(25.2 \%)$ & 0.742 \\
\hline \multicolumn{4}{|l|}{ Comorbid } \\
\hline Yes & $17(17.2 \%)$ & $15(15.1 \%)$ & \\
\hline No & $32(29.3 \%)$ & $35(39.4 \%)$ & 0.725 \\
\hline Distance traveled, $\mathrm{km}$ & $15 \pm 5$ & $178.9 \pm 190.8$ & 0.005 \\
\hline \multicolumn{4}{|l|}{ Social class } \\
\hline High & $09(9.1 \%)$ & $3(3 \%)$ & \\
\hline Middle & $27(27.3 \%)$ & $30(30.3 \%)$ & \\
\hline Low & $13(13.1 \%)$ & $17(17.2 \%)$ & 0.856 \\
\hline \multicolumn{4}{|l|}{ ER presentation } \\
\hline Trauma call & $13(13.01 \%)$ & $6(6.1 \%)$ & \\
\hline RTA & $8(8.1 \%)$ & $5(5.1 \%)$ & \\
\hline Gunshot & $5(5.1 \%)$ & $1(1.1 \%)$ & \\
\hline Consults & $36(36.4 \%)$ & $44(44.4 \%)$ & \\
\hline IO & $6(6.2 \%)$ & $8(8.2 \%)$ & \\
\hline Cholecystitis & $7(7.1 \%)$ & $3(3.1 \%)$ & \\
\hline Pancreatitis & $7(7.1 \%)$ & $2(2.1 \%)$ & \\
\hline Lower GI bleed & $3(3.1 \%)$ & $4(4.1 \%)$ & \\
\hline Nonspecific AP & $1(1.1 \%)$ & $6(6.1 \%)$ & \\
\hline Gangrenous foot & $1(1.1 \%)$ & $5(5.5 \%)$ & \\
\hline Pneumoperitoneum & $2(2.2 \%)$ & $3(3.1 \%)$ & \\
\hline Acute appendicitis & $1(1.1 \%)$ & $4(4.2 \%)$ & \\
\hline Others & $8(8.2 \%)$ & $13(13.1 \%)$ & \\
\hline
\end{tabular}

$E D$ emergency department, $R T A$ road traffic accident, $I O$ intestinal obstruction, GI gastrointestinal, $A P$ abdominal pain

$(10.1 \%)$, acute pancreatitis $(9.1 \%)$, and road traffic accidents $(6.2 \%)$ were among the most common reasons for presentation. Forty-two patients $(42.5 \%)$ were operated on at AKUH. Of those patients, six were taken immediately to the operating room (OR) for treatment of gunshot injuries. Forty-nine patients $(49.5 \%)$ were transferred from another facility to AKUH, and the most common reasons were lack of satisfaction with surgical care $(n=42,85.7 \%)$ and non-availability of surgical personnel $(n=7,14.1 \%)$. Among transferred patients, $71.4 \%(n=35)$ had one transfer, and $28.5 \%(n=14)$ had two transfers. The mean transfer time was $27 \mathrm{~h}( \pm 112 \mathrm{~h})$. Patients came from various locations throughout the country (Fig. 1), and the mean distance traveled was $178 \mathrm{~km}( \pm 190 \mathrm{~km})$ (Table 1).

The mean total wait time in the ED was $8.3 \mathrm{~h}( \pm 4 \mathrm{~h})$. The mean time from registration to generation of a surgery consult was $1.6 \mathrm{~h}( \pm 1.3 \mathrm{~h})$. Mean time for hospital admission was $2.7 \mathrm{~h}( \pm 2.2 \mathrm{~h})$. In 56 cases there was a

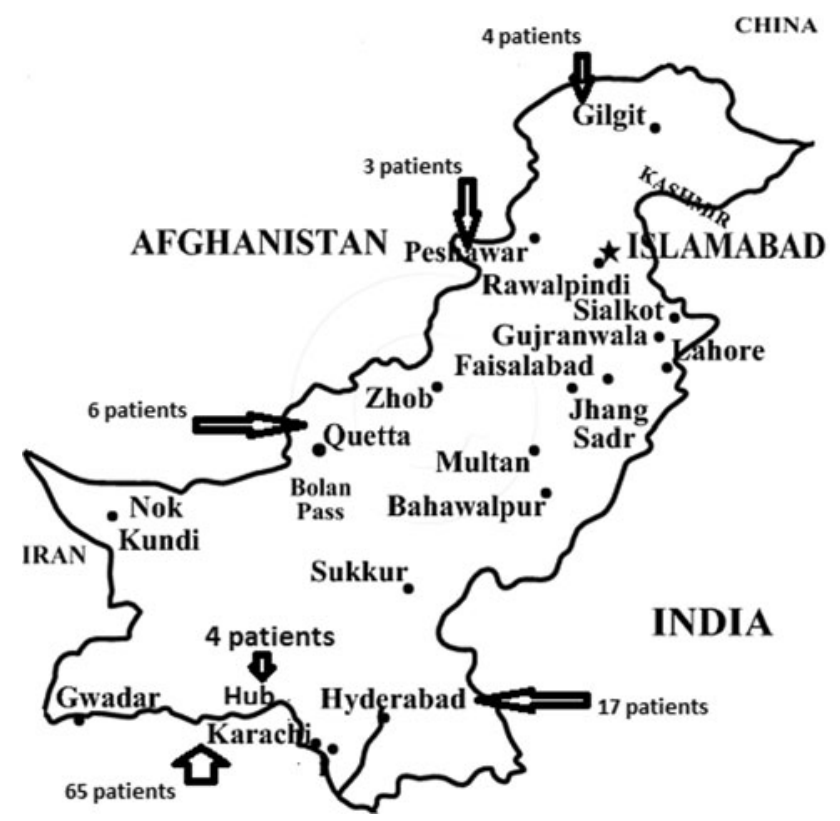

Fig. 1 Patients presenting to Aga Khan University Hospital (AKUH) from all over Pakistan, with number of patients from each city

delay in admission (a greater than $4 \mathrm{~h}$ wait after the decision for admission was made). Common reasons for this delay were bed availability, followed by financial constraints. Three patients were transferred out of the hospital because no ventilator was available, and 14 patients left against medical advice (LAMA) because of financial constraints. One patient died in the ER; he had severe sepsis, most likely exacerbated by delays (Fig. 2).

There were significant differences in mean pulse on arrival (115.9 bpm vs. $96.84 \mathrm{bpm} ; p<0.001)$, oxygen saturation (95 vs. $98 \% ; p<0.001$ ), respiratory rate (32 breaths/min vs. 19 breaths/min; $p<0.001$ ), fluid for resuscitation $(1,958 \mathrm{ml}$ vs. $1,131 \mathrm{ml} ; p<0.001)$, GCS (10 vs. $15 ; p<0.001$ ), revised trauma scoring (TRS) (10 vs. $12 ; p<0.001)$ between transfer and non-transfer patients. Patients who were transferred had poorer vital statistics than those who arrived directly. There was significant difference found in the ICU length of stay (5.67 days vs. 1.5 days, $p=0.022$ ) between the two groups (Table 2).

\section{Discussion}

Surgical care is no longer considered a luxury and has now been incorporated in health care as an essential component [17]. Disparities exist in provision of surgical care worldwide. Low and middle income counties (LMIC) make up $70 \%$ of world population where only $25 \%$ of global surgeries are performed annually [18]. The root cause of this significant inequality is lack of trained surgical staff 
Fig. 2 Flow of patients presented to the AKUH emergency department (ED)

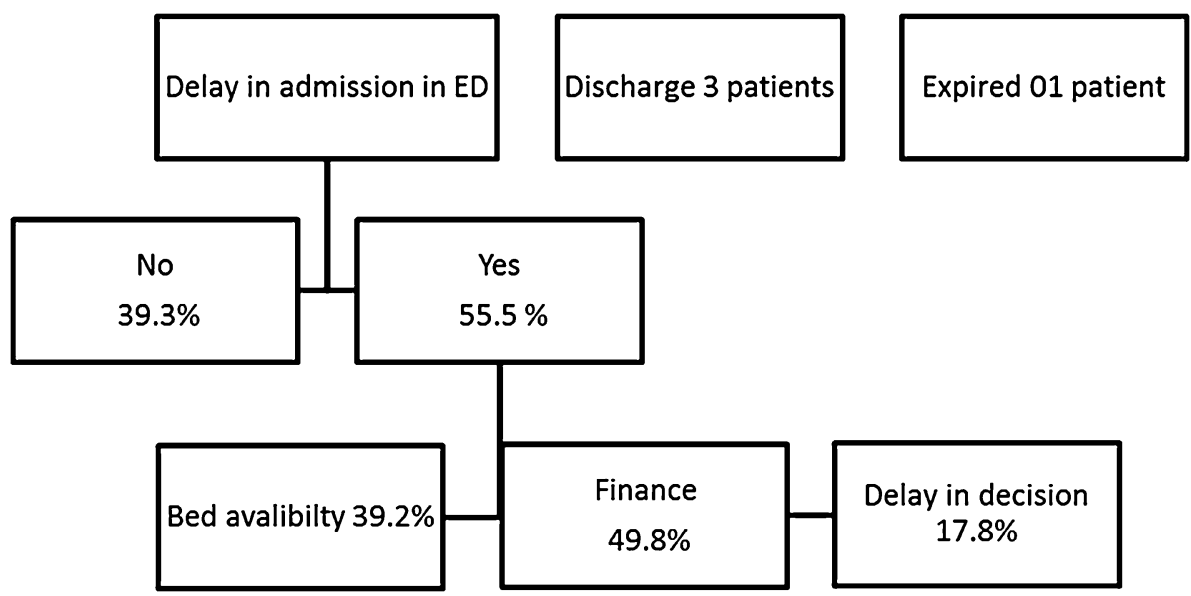

Table 2 Subjective and objective assessment of transferred and nontransferred patients

\begin{tabular}{|c|c|c|c|}
\hline Variable & $\begin{array}{l}\text { Transfer } \\
(n=49)\end{array}$ & $\begin{array}{l}\text { Non-transfer } \\
(n=50)\end{array}$ & $p$ value \\
\hline \multicolumn{4}{|l|}{ Subjective assessment } \\
\hline \multicolumn{4}{|l|}{ Change in ASA } \\
\hline Yes & $24(92.3 \%)$ & $2(7.7 \%)$ & \\
\hline No & $25(43.2 \%)$ & $48(65 \%)$ & 0.000 \\
\hline \multicolumn{4}{|l|}{ Change in procedure } \\
\hline Yes & $17(34.7 \%)$ & $2(4 \%)$ & \\
\hline No & $32(65.3 \%)$ & $48(96 \%)$ & 0.000 \\
\hline \multicolumn{4}{|l|}{ Objective assessment } \\
\hline \multicolumn{4}{|l|}{ Pulse, beats/min } \\
\hline On arrival & 116 & 97 & \\
\hline After resuscitation & 100 & 87 & 0.000 \\
\hline Oxygen saturation & $95 \%$ & $98 \%$ & 0.000 \\
\hline \multicolumn{4}{|l|}{ Respiratory rate } \\
\hline Breaths/min & 33 & 19 & 0.000 \\
\hline Fluid resuscitation, ml & 1,958 & 1,131 & 0.000 \\
\hline GCS (RTA only) & 10 & 15 & 0.000 \\
\hline RTS (RTA only) & 10 & 12 & 0.003 \\
\hline \multicolumn{4}{|l|}{ Invasive monitoring } \\
\hline Yes & $23(46.9 \%)$ & $2(4 \%)$ & 0.000 \\
\hline No & $26(53 \%)$ & $48(96 \%)$ & \\
\hline \multicolumn{4}{|l|}{ Hospital stay, days } \\
\hline ICU & 5.67 & 1.5 & 0.022 \\
\hline SCU & 7.12 & 3.3 & 0.066 \\
\hline Ward & 8.9 & 4.7 & 0.057 \\
\hline
\end{tabular}

ASA American Society of Anesthesiologists score, GCS Glasgow coma scale, RTS revised trauma score, ICU intensive care unit, $S C U$ special care unit

and access to surgical care in low- and middle-income countries (LMIC). Africa has been the focus of a number of studies that identify the surgical needs and practices of various humanitarian and nongovernmental organizations
(NGOs) [18-23]. Tremendous efforts by international bodies have brought issues of access to surgical care in Africa to the global health agenda [24]. International involvement in Africa has also brought about basic changes in the distribution of trained surgical personnel by NGOs leading to improvements in the basic health infrastructure [24]. This study will also help bring Pakistan to the world's attention, focusing on surgical inadequacies and the consequences for the Pakistani people.

Pakistan is among the areas in the world where facilities for essential surgical care are scarce, especially in remote and rural areas. Pakistan is the sixth most populous country in the world, having a population of 160 million, the majority $(65 \%)$ residing in rural areas, and a literacy level touching $46 \%$. The doctor-population ratio approaches 1:1,326 [25]. Making the situation more grave, trained surgical staff is also lacking in most of the primary and secondary care hospitals. This results in estimated 187 deaths per 100,000 population per year from preventable surgical condition [17]. Unlike most African countries, prominent NGOs and WHO have not put in any significant effort to identify the burden of surgical disease, the provision of surgical care, and the effects of surgical transfer in Pakistan.

Surgical care is extremely disappointing in Pakistan [17]. Because trained surgical staff are almost nonexistent at district level hospitals, inter-facility transfer is commonplace and leads to delays in diagnosis. This scenario is routine in Pakistan, and the result is that critically ill surgical patients require prolonged and more complex treatment with significant utilization of resources. In this study we found that surgical transfers are frequent. The most common reasons for transfer were lack of satisfaction with hospital services on the part of patients and their families and, oftentimes, non-availability of basic surgical facilities. Inter-facility transfer leads to delayed institution of appropriate treatment and poor patient outcomes. A study from Northern Pakistan has concluded that poor outcomes in surgically treatable patients were due to misinterpretation of 
symptom severity by first level heath care providers and late referral to an appropriate surgical facility [7, 9]. Patients in developed countries face the same situation; in a study from Ohio, the rate of perforated appendix was higher in rural areas with inadequate access to a metropolitan hospital [17]. In contrast, Mbah et al [13] in 2006 studied all patients who presented with acute surgical abdomen in a Nigerian teaching hospital and observed that poverty is the major hindrance to seeking surgical advice, and this often results in delayed presentation. With $98 \%$ of the Pakistani population belonging to low and middle social class, the scenario in Pakistan is not very different [25].

An established ED is essential for timely and accurate surgical care, and is correctly called the "safety net" of any health care system [26]. Emergency Department overcrowding is a worldwide problem that affects the ability to provide timely surgical care and therefore outcome [26, 27]. Compared to shorter ED stay, a prolonged length of stay in the ED is associated with poor outcome [19, 20]. In developed countries, high hospital bed occupancy is responsible for a longer ED stay, whereas in developing countries financial constraints are the most common reason $[27,28]$. In the present study the patients had to wait in the ED pending arrangements for covering the cost of treatment, which resulted in further delay and worsening of patients' physiology. Another important delaying factor in our study was bed availability, which is a recognized problem worldwide. Multiple efforts have been made by different countries to shorten the ED waiting time by establishing buffers to empty inpatient beds, improve the acute admission process, add an express admit unit, and establish an expedited admission protocol [26-28].

In an attempt to decrease ED overcrowding and prolonged waiting time, we have started a critical decision unit (CDU) to observe stable patients for 24 to $48 \mathrm{~h}$. This helps reduce waiting time and leads to release of patients from the ED once they are ready for discharge.

Our study was of short duration with small sample size, but it is the first study addressing surgical transfers in Pakistan. Moreover, the objective parameters of transferred and non-transferred patients were measured prospectively, something that has not been monitored in reports in the international literature. We believe this will further motivate international organizations to obtain measurable data and streamline the process of surgical care provision to the neediest areas of the world.

Subjectivity of the study in terms chief resident's input is also a limiting factor, but we overcame this by adding objective parameters for assessment. In this study patients who had two inter-facility transfers $(28 \%)$ experienced more deterioration of their clinical condition. To date no such finding has been reported in literature. We also determined that distances traveled by transferred and nontransferred patients, had not been reported in any previous study.

This study also brings out that distribution of trained surgical personnel should always be needs based. Surgical staff should be strategically placed across the board to replenish the shortages and treat different problems at their respective hospital levels, thereby reducing the burden on tertiary care facilities. Policy makers should include surgical care in the planning of district health systems, as the provision of surgical care is the most cost effective component. In addition, the ambulance system needs to be organized and adequately equipped to transfer patients to high level surgical care units. Finally, an expedited admission protocol and a long stay ward are necessary to decrease ED waiting time.

This study is first of its kind from this region that has identified measurable outcomes of delayed transfer, because the international literature is lacking in this respect. It will also provide a platform from which to conduct better designed research regarding surgical care provision and utilization, while addressing unmet surgical needs. The findings of this study may also bring Pakistan to the world's attention in general, and to international humanitarian organizations in particular, as an area where inadequacies in surgical care affect delivery of healthcare.

\section{References}

1. Taira BR, Kelly McQueen KA, Burkle FM Jr (2009) Burden of surgical disease: does the literature reflect the scope of the international crisis? World J Surg 33:893-898. doi:10.1007/ s00268-009-9981-x

2. Ozgediz D, Jamison D, Cherian M et al (2008) The burden of surgical conditions and access to surgical care in low- and middle-income countries. Bull World Health Organ 86:646-647

3. Ozgediz D, Hsia R, Weiser T et al (2009) Population health metrics for surgery: effective coverage of surgical services in low-income and middle-income countries. World J Surg 33:1-5. doi:10.1007/s00268-008-9799-y

4. Atiyeh BS, Gunn SW, Hayek SN (2010) Provision of essential surgery in remote and rural areas of developed as well as low and middle income countries. Int J Surg 8:581-585

5. Young JS, Bassam D, Cephas GA et al (1998) Interhospital versus direct scene transfer of major trauma patients in a rural trauma system. Am Surg 64:88-91 discussion 91-92

6. Holena DN, Mills AM, Carr BG et al (2011) Transfer status: a risk factor for mortality in patients with necrotizing fasciitis. Surgery $150: 363-370$

7. Ahmed M, Shah M, Luby S et al (1999) Survey of surgical emergencies in a rural population in the Northern Areas of Pakistan. Trop Med Int Health 4:846-857

8. Waseem H, Naseer R, Razzak JA (2011) Establishing a successful pre-hospital emergency service in a developing country: 
experience from rescue 1,122 service in Pakistan. Emerg Med J 28:513-515

9. Razzak JA, Hyder AA, Akhtar T et al (2008) Assessing emergency medical care in low income countries: a pilot study from Pakistan. BMC Emerg Med 8:8

10. Razzak JA, Cone DC, Rehmani R (2001) Emergency medical services and cultural determinants of an emergency in Karachi, Pakistan. Prehosp Emerg Care 5:312-316

11. Khan A, Zafar H, Naeem SN et al (2010) Transfer delay and inhospital mortality of trauma patients in Pakistan. Int J Surg 8:155-158

12. Blanchard RJ, Blanchard ME, Toussignant $P$ et al (1987) The epidemiology and spectrum of surgical care in district hospitals of Pakistan. Am J Public Health 77:1439-1445

13. Mbah N, Opara WEK, Agwu NP (2006) Waiting time among acute abdominal emergencies in a Nigerian teaching hospital: causes of delay and consequences. Nigerian J Surg Res 8:35

14. Galante JM, Phan HH, Wisner DH (2010) Trauma surgery to acute care surgery: defining the paradigm shift. J Trauma 68:1024-1031

15. Sheikh L, Najmi N, Khalid U et al (2011) Evaluation of compliance and outcomes of a management protocol for massive postpartum hemorrhage at a tertiary care hospital in Pakistan. BMC Pregnancy Childbirth 11:28

16. Komashie A, Mousavi A (2005) Modeling emergency departments using discrete event simulation techniques. Paper presented at the Proceedings of the 37th conference on Winter Simulation

17. Zafar SN, Fatmi Z, Iqbal A et al (2013) Disparities in access to surgical care within a lower income country: an alarming inequity. World J Surg 37:1470-1477

18. Petroze RT, Nzayisenga A, Rusanganwa V et al (2012) Comprehensive national analysis of emergency and essential surgical capacity in Rwanda. Br J Surg 99:436-443
19. Chao TE, Burdic M, Ganjawalla K et al (2012) Survey of surgery and anesthesia infrastructure in Ethiopia. World $\mathrm{J}$ Surg 36:2545-2553. doi:10.1007/s00268-012-1729-3

20. Henry JA, Windapo O, Kushner AL et al (2012) A survey of surgical capacity in rural southern Nigeria: opportunities for change. World J Surg 36:2811-2818

21. Lavy C, Sauven K, Mkandawire N et al (2011) State of surgery in tropical Africa: a review. World J Surg 35:262-271

22. Ozgediz D, Galukande M, Mabweijano J et al (2008) The neglect of the global surgical workforce: experience and evidence from Uganda. World J Surg 32:1208-1215. doi:10.1007/s00268-0089473-4

23. Grimes CE, Law RS, Borgstein ES et al (2012) Systematic review of met and unmet need of surgical disease in rural sub-Saharan Africa. World J Surg 36:8-23

24. Spiegel DA, Abdullah F, Price RR et al (2013) World Health Organization global initiative for emergency and essential surgical care: 2011 and beyond. World J Surg 37:1462-1469. doi:10. 1007/s00268-012-1831-6

25. Nishtar S (2010) Choked pipes-reforming Pakistan's mixed health system. J Pak Med Assoc 60:252-253

26. Buckley BJ, Castillo EM, Killeen JP et al (2010) Impact of an express admit unit on emergency department length of stay. J Emerg Med 39:669-673

27. Moloney ED, Bennett K, O'Riordan D et al (2006) Emergency department census of patients awaiting admission following reorganisation of an admissions process. Emerg Med J 23:363-367

28. Proudlove NC, Gordon K, Boaden R (2003) Can good bed management solve the overcrowding in accident and emergency departments? Emerg Med J 20:149-155 